\title{
eNeuro
}

Research Article: New Research / Cognition and Behavior

\section{Reward value enhances sequence monitoring ramping dynamics as ending rewards approach in the rostrolateral prefrontal cortex}

https://doi.org/10.1523/ENEURO.0003-22.2022

Cite as: eNeuro 2022; 10.1523/ENEURO.0003-22.2022

Received: 3 January 2022

Accepted: 25 January 2022

This Early Release article has been peer-reviewed and accepted, but has not been through the composition and copyediting processes. The final version may differ slightly in style or formatting and will contain links to any extended data.

Alerts: Sign up at www.eneuro.org/alerts to receive customized email alerts when the fully formatted version of this article is published. 4.0 International license, which permits unrestricted use, distribution and reproduction in any medium provided that the original work is properly attributed. 
Title: Reward value enhances sequence monitoring ramping dynamics as ending rewards approach in the rostrolateral prefrontal cortex

\section{Abbreviated Title: Reward enhances sequence ramps in RLPFC}

Theresa H. McKim ${ }^{1}$ and Theresa M. Desrochers ${ }^{1,2,3^{*}}$

${ }^{1}$ Department of Neuroscience, Brown University, Providence, RI, 02912, USA

${ }^{2}$ Department of Psychiatry and Human Behavior, Brown University, Providence, RI, 02912, USA

${ }^{3}$ Robert J. and Nancy D. Carney Institute for Brain Science, Brown University, Providence, $\mathrm{RI}, 02912$, USA

Author Information:

Dr. Theresa H. McKim, Department of Neuroscience, Brown University, Box GL-N, Providence, RI 02912.

* Correspondence should be addressed to: Dr. Theresa M. Desrochers, Department of Neuroscience, Brown University, Box GL-N, Providence, RI 02912. E-mail:

theresa desrochers@brown.edu

Number of pages: 29

Number of figures: 5

Number of tables: 3

Number of words

Abstract: 247

Significance: 119

Introduction: 776

Discussion: 1,035

Conflict of interest statement: The authors declare no competing financial interests or other conflicts related to the text of this paper.

Acknowledgements:

We would like to thank Adriane Spiro, Nadira Yusif Rodriguez, Debaleena Basu, Matthew Maestri, Hannah Doyle, and Vivian Lu for their assistance with data collection and preparation of this article. We also thank Aarit Ahuja, Katie Conen, Debbie Yee, and members of the Desrochers Lab for many helpful discussions. Research reported in this publication was supported by a NIDA F32 DA045451 (Theresa H. McKim), and Brown University Department of Neuroscience Connors Fellowship (Theresa H. McKim), and NIGMS COBRE (P20GM103645, Theresa M. Desrochers). Part of this research was conducted using computational resources and services at the Center for Computation and

43 Visualization, Brown University, NIH grants S10 OD016366 and S10 OD025181. 


\section{4}

\section{Introduction}

78 A hallmark of goal-directed behavior in everyday life is the performance of sequential tasks

\section{Abstract}

Many fundamental human behaviors contain multiple sequences performed to reach a desired outcome, such as cooking. Reward is inherently associated with sequence completion and has been shown to generally enhance cognitive control. However, the impact of reward on cognitive sequence processing remains unexplored. To address this key question, we focused on the rostrolateral prefrontal cortex (RLPFC). This area is necessary and exhibits increasing ("ramping") activation during sequences, a dynamic that may be related to reward processing in other brain regions. To separate these dynamics, we designed a task where reward was only provided after multiple four-item sequences ("iterations"), rather than each individual sequence. Using fMRI in humans, we investigated three possible interactions of reward and sequential control signals in RLPFC: (1) with the visibility of sequential cues, i.e. memory; (2) equally across individual sequence iterations; and (3) differently across individual sequence iterations (e.g., increasing as reward approaches). Evidence from previous, non-sequential cognitive control experiments suggested that reward would uniformly change RLPFC activity across iterations and may depend on the visibility of cues. However, we found the influence of reward on RLPFC ramping increased across sequence iterations and did not interact with memory. These results suggest an active, predictive, and distinctive role for RLPFC in sequence monitoring and integration of reward information, consistent with extant literature demonstrating similar accelerating reward-related dopamine dynamics in regions connected to RLPFC. These results have implications for understanding sequential behavior in daily life, and when they go awry in disorders such as addiction.

\section{Significance Statement}

We daily perform multiple sequences to achieve rewarding goals, but little is known about how control of these sequences and reward information interacts in the brain. The rostrolateral prefrontal cortex (RLPFC) is necessary to perform sequential tasks and contains reward-related signals, making it a key potential site of integration. We designed a human fMRI experiment to test three possibilities for how reward and sequential control processes may interact: (1) through a non-sequential process; (2) uniformly across sequences; (3) non-uniformly across sequences. We found that reward shows greater interaction with sequential control dynamics in the RLPFC as the ending reward approaches. These results provide insight into the function of RLPFC and how rewards are integrated into temporally extended control processes. to reach a reward. For example, when cooking, a series of tasks, such as chopping, adding ingredients, and stirring, must be monitored and executed before sitting down to a satisfying meal. Completing these tasks requires cognitive control over items in the progression ("sequential control"). Progress has been made in understanding how rewards generally enhance non-sequential cognitive control behaviors and neural dynamics (Braver et al., 
2014; Botvinick and Braver, 2015; Yee and Braver, 2018; Chiew, 2021), but how rewards may modulate sequential control processes remains an open question.

Sequential control and reward both underlie dynamics in the rostrolateral prefrontal cortex (RLPFC, also referred to as anterior prefrontal cortex or lateral frontal polar cortex). Human fMRI and transcranial magnetic stimulation (TMS) experiments have shown that RLPFC is necessary for sequential control and monitoring (Desrochers et al., 2015, 2019). In nonsequential tasks, RLPFC tracks reward trends (Kovach et al., 2012), demonstrates increased task-related activity with increased reward (Pochon et al., 2002), and correlates with individual differences in reward activation (Locke and Braver, 2008). Thus, RLPFC is an ideal initial focus for examining dynamics in a network integrating reward information with sequential control.

The RLPFC also shares key connections and similarities in dynamics with regions and reward-related neurotransmitters. In this area, activity increases ("ramps") from the first to the last position of the sequence (Desrochers et al., 2015, 2019). Other reward-related brain regions such as the anterior cingulate cortex (ACC), striatum, and ventral tegmental area (VTA) show ramping neural activity as rewards approach (Totah et al., 2013; Ma et al., 2014; Wang et al., 2018) and dopamine concentration increases (Howe et al., 2013; Hamid et al., 2015). RLPFC is anatomically connected to these (Desrochers and Badre, 2012; Haber and Behrens, 2014; Coenen et al., 2018) and other reward processing regions such as the orbitofrontal cortex (Thiebaut de Schotten et al., 2012) and ventromedial prefrontal cortex (Haber and Behrens, 2014). Similar dynamics in RLPFC and connected rewardrelated regions further raises the potential for interaction of processing-related activity within RLPFC.

Typically, the influence of reward is studied in association with a single action or event. In the less frequent situation where reward is studied in conjunction with sequences, it often occurs at the end of a single sequence, making the effects of reward and sequence completion inseparable. Therefore, to disentangle the effects of reward and sequence on RLPFC activity we designed a task, described in further detail below, that required participants to complete multiple four-item sequences before receiving reward. We term a single instance of a four-item sequence as an "iteration". Participants performed several iterations of the same sequence (e.g., cutting up multiple items while cooking) to obtain reward. With this paradigm, we examined the interaction between reward and sequence processing signals in the RLPFC.

Reward and sequential control dynamics could interact in several ways across multiple sequence iterations. First, reward effects could be uniform but nonspecific to sequence iterations. For example, several memory processes influenced by reward have been localized to RLPFC (Shallice et al., 1994; Christoff et al., 2001; Braver and Bongiolatti, 2002; Shigemune et al., 2017)1/27/2022 10:07:00 AM. Second, reward may have a uniform influence across items within individual sequences. In non-sequential tasks, RLPFC exhibits tonic (Beck et al., 2010) and increasing (Chiew et al., 2016) reward-related activity changes. 
Third, reward may have an influence that is non-uniform across sequence iterations (e.g., increasing as reward approaches). Serial behaviors (Braun et al., 2018) and computational modeling (Ott et al., 2020) suggest that reward information representation changes as temporally distant rewards approach. Further, dopamine concentrations in the striatum show accelerating changes as reward approaches (Howe et al., 2013), but only in regions directly involved in task completion (Hamid et al., 2021). Thus, the nature and distribution of potential interactions has consequences for how RLPFC controls sequential processes in the presence of reward.

To test these alternatives, human participants monitored multiple sequence iterations under differing reward and cue visibility conditions to manipulate memory demand. Based on reward influence on non-sequential cognitive control (Beck et al., 2010; Chiew et al., 2016), we hypothesized that reward would change RLPFC sequence monitoring dynamics uniformly across individual sequences. We found that reward did not change the slope or magnitude of individual sequence ramping or interact with memory demand in RLPFC. Instead, we provide novel evidence that reward interacts with sequential dynamics only as the end reward approaches. These results suggest that non-linear increases in activity proximal to reward outcome realization is important for assigning value to temporarily extended cognitive control processes, and further implicate RLPFC as a crucial node in processing abstract sequence information.

\section{Materials and Methods}

\section{Participants}

Thirty-six ( $n=20$ female) adults between the ages of 18-35 years of age $(M=24 ; S D=5)$ were included for analysis in the experiment. An additional 18 participants performed the task but were excluded from analyses due to poor participant performance, poor data quality, and equipment malfunction issues and are detailed as follows: Five participants were unable to complete the task and/or fell asleep. One participant had a previously undetected brain abnormality and was subsequently excluded from analysis. One participant had very low signal to noise ratio (SNR), and four participants had excessive head motion $(>3 \mathrm{~mm})$. Post-hoc it was discovered that seven participants completed the task when the response button box began malfunctioning (MR compatible four button response pad, Mag Design and Engineering). This malfunction resulted in an inability to dissociate participant error from response box malfunction and a large number of trials that had to be excluded for incorrect responses $(>30 \%)$. Therefore, we excluded these seven participants and the button box was replaced with a fiber optic response pad ( 5 button handheld device, Cambridge Research Systems) for the remaining participants. We confirmed there were no differences in reaction times between participants based on the response device for all behavioral analyses reported below (all F's $<3.92$, p's $>0.07$ ).

All participants were right-handed, had normal or corrected-to-normal vision, and reported they were not colorblind. Individuals with neurological or psychiatric conditions, brain injury, 
or reported use of psychoactive medications or substances were excluded from participating. Participants were recruited from the Brown University campus and the surrounding community. Participants were compensated $\$ 20 / \mathrm{hr}$ for their time. Additionally, participants received up to a $\$ 10$ bonus based on task performance. Specifically, participants were instructed prior to scanning that one run would be randomly selected after task completion to determine the performance bonus. This instruction was used to motivate and encourage task performance across all scanning runs. In reality, all participants received a $\$ 10$ bonus for task completion. All participants gave informed, written consent as approved by the Human Research Protections Office at Brown University.

\section{$\underline{\text { Task Design and Procedure }}$}

\section{Overview}

Participants completed a modified version of the sequence monitoring task published in Desrochers et al., (2019). Participants monitored serially presented four-item sequences and for each item, indicated whether it was in or out of the pre-instructed order with a button press (FIG 1). Each participant completed a single session that included task training, a short sequence preference test, task performance while undergoing fMRI scanning, and post-scanning sequence preference test and questionnaires.

\section{Trial Structure}

Sequences were composed of four unique visual stimuli drawn from a pool of common objects (Desrochers et al., 2019). Four image sets were drawn randomly for each participant. Task stimuli were displayed using an Apple computer running macOS. Experiment scripts were programmed using the Psychophysics Toolbox in Matlab (MathWorks; RRID:SCR_ 001622). On each trial, a stimulus was displayed in the center of the screen on a grey background. Participants were asked to press a button to indicate whether the displayed image was in or out of the instructed sequence order while the image was presented on the screen for 1 second. An intertrial interval (ITI) followed each image and displayed a fixation cross centrally. The ITI timing was jittered and optimized across trials for scanning $(0.25-8 \mathrm{~s}$, mean $2 \mathrm{~s})$.

Participants responded with the index and middle fingers on their right hand. One key was assigned the 'in sequence' response and the other was the 'out of sequence' response. Response options were counterbalanced across participants. Participants were instructed to respond as quickly and accurately as possible while the stimulus was displayed on the screen (1 s). Occasional responses (mean $8 \%$, across participants) that occurred in the ITI were included in analyses to avoid unnecessary data loss.

\section{Block Structure}

Rewards were infrequent in the task structure because participants completed multiple fouritem sequences before obtaining reward. Therefore, we adopted a nested block structure to maximize the number of sequence and reward trials within a reasonable amount of time for 
the participant to perform the task in the scanner ( $1 \mathrm{hr})$. This nested structure saved time by allowing two blocks to be completed with only one instruction period. An overview of the terminology and structure follows:

- Condition: One of the four possible combinations of the two-reward by two-visibility type design: Visible High, Visible Low, Occluded High, Occluded Low (Fig. 1A-D).

- Image set: One of four pre-learned sets of image orders (see Training). Each image set was only associated with either high or low value. However, each image set was used for both Occluded and Visible trials (Fig. 1D).

- Hyperblock: Two, consecutive blocks of the same condition (Fig. 1C). Consecutive hyperblocks would not be the same condition, as the order was randomized (Fig. 1E).

- Block: a group of monitoring trials, probe, and reward (Fig. 1A-C).

- Red screen: Signal to get ready at the beginning of the first block of a hyperblock, and that a new condition was starting (Fig. 1A-C).

- Instruction images: Only occurred in the first block of a hyperblock after the red screen. The four images from one image set are displayed, in order, for $0.75 \mathrm{~s}$ each with no time between images. The participant does not respond to these images (Fig. 1A-C).

- Green screen: Signal to get ready at the beginning of the second block of a hyperblock. Because it was the same condition as the first block, no value cue was shown, no instruction images followed it, and participants pressed the button to proceed immediately to monitoring trials (Fig. 1A-C).

- Monitoring trials: Participants responded to indicate whether each image was in or out of sequence as described in Trial Structure for a total of 13-16 consecutive trials (Fig. 1A-C). In the Visible condition, these images were all from the image set, and in the Occluded condition all images were the placeholder image. Monitoring trials can be further grouped into:

- Sequence Iterations: One set of four items that made up the instructed sequence (Fig. 1C). All iterations within a block were from the same image set.

- Iteration 1: Trials 1-4 were not different from subsequent monitoring trials for the participant, but we excluded them from behavior and fMRI analyses to avoid block initiation effects.

- Iteration 2: Trials 5-8. The first iteration used in analysis.

- Iteration 3: Trials 9-12. The second iteration used in analysis.

- Probe trial: The probe trial occurred, with equal probability, during the fourth iteration on trial $13,14,15$, or 16 . In other words, the probe trial occurred with equal probability across the four positions (1-4) in the sequence iteration. Probe trials did not occur at any other place in the block, and only the probe trial could be out of order ( $50 \%$ of the time). In the Occluded conditions, this probe trial image was one of the instructed image set, rather than the occluder image. In the Visible condition, an image from the same set as in the preceding monitoring trials could be displayed out 
of order. As in the monitoring trials, participants must respond to whether the probe image is in or out of sequence.

- Feedback and Reward: Displayed (0.75 s) after the probe trial to indicate whether the participant's response was correct (check mark) or not ("X"). The value of the condition was also displayed as "\$" (low) or “\$\$" (high) on correct trials.

A single hyperblock was composed of two blocks of the same condition. The order of events in a hyperblock was as follows. The first block of a hyperblock began with a red screen that displayed the value. Participants pressed the assigned 'in sequence' key to initiate the block (i.e., the block did not proceed without this response). After initiation, the red screen was followed by the four instruction images from a single image set, the monitoring trials (iterations 1-3), the probe trial during iteration 4, and then the feedback and reward. Between the first and second block of a hyperblock, a fixation cross was displayed for a variable ITI $(0.25-8 \mathrm{~s})$ before the green screen appeared. Participants pushed the in sequence' button to start this second block, which proceeded immediately to the monitoring trials (iterations 1-3), the probe trial during iteration 4 , and then the feedback and reward. A fixation cross was displayed during a variable ITI $(0.25-8 \mathrm{~s})$ before the start of the next hyperblock. For all blocks, if participants responded incorrectly on a monitoring trial prior to the probe trial (e.g., pressing the out of sequence key prematurely), the block was terminated: no further images and incorrect feedback ("X") was immediately displayed.

\section{Run Structure}

During scanning, participants completed six runs of the task. Each run lasted approximately six minutes and contained eight hyperblocks (sixteen total blocks). Each run included two hyperblocks of each of the four conditions (Occluded High, Occluded Low, Visible High, Visible Low). The two hyperblocks of each condition within each run used different image sets (e.g., Visible High Set 1 and Visible High Set 2; Fig. 1D). The order of the hyperblocks was randomized such that consecutive hyperblocks were not of the same condition and were counterbalanced across the six runs (Fig 1E). In between runs, the scanner was stopped, and participants had the option of taking a brief break. Participants' error rate on the previous run was displayed on the break screen to encourage correct performance.

\section{Training}

Participants were trained on the order of the four image sets immediately prior to scanning. During training ( 45 mins), participants learned the reward value (' $\$$ ' = low, ' $\$ \$$ ' = high) and the correct ordering of the four images in each set by completing trials of the task. The meaning of the dollar signs was intentionally abstract to avoid participants explicitly "tallying" reward amounts across trials or blocks. Participants were instructed that they could earn a bonus of up to $\$ 10$ based on their performance on a single run chosen at random, but they were not instructed a direct correspondence between dollar signs and bonus payment amount. We ensured that participants nevertheless understood and used the values associated with the sequences by training them before scanning and using a preference test described in further detail below. 
To learn the correct order of the four image sets used for each participant, participants first completed the task without time constraints. The first time a new sequence was introduced, participants viewed the images serially without a response deadline, i.e., the image remained on the screen until a button press was made. Participants responded with the " $\mathrm{j}$ " and " $k$ " keys on the keyboard to select a response with the index and middle finger of their right hand. Participants practiced two blocks with response times recorded, similar to the actual experiment. Training was conducted on the visible condition of a sequence first, followed by practice of the occluded version. Participants then completed two blocks of occluded trials with response times recorded. This was repeated for each of the four sequences. Training was completed twice for each participant before moving on to completing the task in the scanner.

After training, participants completed a sequence preference test where they were instructed to select the high value sequence of each pair presented. Two of the four ordered image sets were displayed at a time: one set of four images across the top and one image set across the bottom of the screen relative to a central fixation spot. The image sets remained on the screen until one of the image sets was chosen with a button press (i.e., no response deadline) and there was a $1 \mathrm{~s}$ ITI between trials. All 24 combinations of image set pairs were displayed, and the location on the screen was counterbalanced across pairs and presentations. These trials were used to verify that participants had learned the associated reward value of the image sets.

After scanning, participants again completed the sequence preference test to verify that they had retained the learned image set reward values. A post task questionnaire was administered to determine any factors that may have impacted performance and experience with the task during scanning (fatigue/sleep, sequence difficulty, response deadline, etc.), participants were debriefed, and compensated for their time.

\section{Experimental Design and Statistical Analysis}

\section{fMRI data acquisition and preprocessing}

A Siemens 3T PRISMA MRI scanner with a 64-channel head coil was used for whole-brain imaging. Functional data were acquired using a fat saturated gradient-echo, echo planar imaging (EPI) pulse sequence (repetition time, TR=2 s; echo time, $T E=28 \mathrm{~ms}$; flip angle 90 degrees; 38 interleaved axial slices; $3 \times 3 \times 3 \mathrm{~mm}$ ). Anatomical scans included a T1-MPRAGE (TR, $1900 \mathrm{~ms}$; TE, $3.02 \mathrm{~ms}$; flip angle, 9 degrees; 160 sagittal slices; $1 \times 1 \times 1 \mathrm{~mm}$ ) and a T1 in-plane scan (TR, $350 \mathrm{~ms}$; TE $2.5 \mathrm{~ms}$; flip angle, 70 degrees; 38 interleaved transversal slices; $1.5 \times 1.5 \times 3 \mathrm{~mm})$.

Preprocessing and analysis were conducted in SPM12 (http://www.fil.ion.ucl.ac.uk/spm, RRID:SCR_007037). Two participants had one run of data removed due to excessive head motion $(>3 \mathrm{~mm})$. EPI images were slice time corrected and realigned to correct for head motion. Images were normalized to Montreal Neurological Institute (MNI) stereotaxic space 334 and smoothed with an $8 \mathrm{~mm}$ isotropic Gaussian kernel. 


\section{fMRI data analysis}

Subject-specific models of condition effects were constructed in SPM12 under assumptions of the general linear model (GLM). Regressors were generated by convolving events of interest with the canonical hemodynamic response function and included the temporal derivative. If any trial in the block was incorrect or the participant stopped responding to the images, then the entire block was coded as an error because it was unknown whether the participant was correctly performing the monitoring trials. All models include a duration regressor based on the time the participant waited to start the trials at the block start screen (red or green) and an onset regressor modeled as a stick function for the feedback screen. Other conditions of interest are described below for each model. Nuisance regressors for all participants in all models included: instruction images, the first four monitoring trials in a block (iteration 1), error trials, the duration of the probe trial (including reaction time), a run regressor, and six motion parameters (translation and rotation).

For each participant, runs were entered as a single session and the first level was estimated as a fixed effects model. Whole brain estimates of within subject effects were entered into second level random effects analyses. One-sample t-tests were used to test for significance against zero $(p<0.001)$. Results were corrected for multiple comparisons based on wholebrain group effects with extent thresholds set at the cluster level, yielding a familywise error (FWE) correction $(p<0.05)$. Group contrasts are displayed on an inflated MNI canonical brain using Caret software (Van Essen et al., 2012); RRID:SCR_006260).

\section{Five GLMs were estimated from the data as follows:}

1. Sequence position onsets model. To assess the univariate effects of sequence position, cue visibility, and reward, we constructed a model using instantaneous stimulus onset regressors based on the factors of cue visibility (occluded/visible) $x$ reward (high/low) $x$ sequence position (i.e., position within iteration, 1-4). We provide a simplified version of the regressors for the onsets in FIG 2A. All regressors shown were separated by visibility and reward conditions (e.g., a model containing onsets would contain 16 regressors: Occluded High Positions 1-4; Occluded Low Positions 1-4; Visible High Positions 1-4; Visible Low Positions 1-4).

2. Parametric sequence position (ramping) model. This ramping model tests for activation that increases with sequence position (Desrochers et al., 2015, 2019). Onset regressors were constructed by crossing the factors of cue visibility (occluded/visible) $x$ reward (high/low) to result in the four condition combinations. Sequence position (1-4) was added as a parametric modulator of the onsets for the positions. Temporal derivatives of the parametric regressors were also included. The parametric regressors are estimated hierarchically to account for variance above and beyond that explained by the onsets alone. Additionally, we included a separate nuisance regressor for the last image in a block (probe trial) (FIG 2B).

3. Sustain versus unique ramp model. We sought to identify whether variance in the fMRI signal was uniquely explained by sustained versus ramping activation. Models that included both sustained and ramping activation were constructed to allow the regressors to compete for variance within the same model. Sustain and ramp regressors (separate for each combination of cue visibility $\mathrm{x}$ reward) were included in addition to a single regressor for stimulus onset at each position. Sustain and ramp regressors started at stimulus onset of each sequence (position 1) and ended at the 
stimulus offset (button press) to sequence position 4. This initial model was used to identify variance uniquely explained by the ramp regressor. The sustain and ramp regressors were orthogonalized (spm_orth.m) within the condition combinations to remove shared variance from the ramp regressors (and assign it to the sustain regressors) (FIG 2C-D).

4. Unique sustain versus ramp model. This model complements the one above to identify variance uniquely explained by the sustain regressor (independent of ramp). The shared variance from the sustain regressors was removed and assigned to the ramp regressor. This model was used to identify variance uniquely explained by the sustain regressor. All other aspects of the model were the same as the previous sustain versus unique ramp model (FIG 2C-D).

5. Block trial number onsets model. To determine how the fMRI signal evolves throughout the course of trials within a block, we modeled two complete sequence iterations (iterations 2 and 3 ) of each block as individual trials (excluding iteration 1 to avoid block initiation effects, see Block Structure). Regressors were modeled as onsets as in FIG 2A but classified by trial across the block (5-12), for a total of eight regressors, instead of only four regressors for sequence position (1-4) as in Model 1.

a. Individual regressors were included for sequence positions (trials 5-12). Onset regressors were again included as nuisance regressors for the first four trials of the block to remove block initiation effects. For the remaining trials (13-16) in iteration 4 at the end of a block where the probe image could appear, an onset regressor of the same condition for each trial was included as a nuisance effect. All other aspects of the model were the same as Model 1. For the initial model, we collapsed across all conditions to examine overall block dynamics.

b. To examine monitoring without visible position cues, we created a model that included the same regressors above (5a) but included separate regressors for the occluded and visible conditions collapsed across reward conditions.

c. To examine reward influence, we created a model that included the same regressors as above (5a) but included separate regressors for the high and low reward conditions collapsed across visibility conditions.

d. To examine the interaction between monitoring without visible position cues and reward, we created a model that included the same regressors above (5a) but included separate regressors for the combinations of visible/occluded and high/low reward conditions.

\section{Region of interest (ROI) Analysis}

ROls were primarily defined from activation in the parametric ramp $>$ baseline contrast in Desrochers et al. (2019). MNI coordinates for the RLPFC ROI defined by the cluster of activation in the parametric ramp $>$ baseline contrast in Desrochers et al. (2019) are at $x=-$ $36, y=42, z=34$ (center of mass) and will be hereafter referred to as the "D19" ROI. Limited post-hoc supporting analyses were performed using the RLPFC cluster from the same contrast in the present study (across all conditions). The mean beta values were extracted from the parametric ramp regressor across all voxels in the ROI using the Marsbar toolbox (MarsBar SPM toolbox, RRID:SCR_009605) in SPM. Ramping activation across models and regions were compared using repeated measures ANOVA and paired t-tests where appropriate. For the sequence position onsets and block trial number onsets models, the time course of activity across positions was extracted using an eight-timepoint (16 s) 
430 finite impulse response (FIR) model in the MarsBar toolbox in SPM that contained the same

431 regressors as the onset model.

\section{Behavioral analysis}

433 As in previous studies (Desrochers et al., 2015, 2019), we excluded the first four trials of 434 every block (iteration 1) from analysis to remove bias in reaction times (RTs) from block 435 initiation effects (Schneider and Logan, 2006). RT analyses excluded error trials. To match 436 data for examining trials across blocks, responses in the ITI (mean 8\%, across participants), 437 were included to avoid unnecessary data loss. For ER, we also conducted analyses to 438 determine detection of an out of sequence item. We defined detection types as hits, correct 439 rejections, misses, and false alarms to calculate d-prime. Hits were defined as correct 440 responses to an out of sequence item. Correct rejections were correct responses to in 441 sequence trials in both visible and occluded conditions. Misses were button presses 442 indicating in sequence to an out of sequence item. False alarms were out of sequence 443 responses to an in-sequence item. D-prime $\left(d^{\prime}\right)$ was calculated:

$$
\left.d^{\prime}=Z \text { (hit rate }\right)-Z(\text { false alarm rate })
$$

where $Z(p), p \in[0,1]$, is the inverse of the normal cumulative distribution function (Macmillian and Creelman, 2004). Extreme rates of zero or one were converted to $1 /(2 \mathrm{M})$ and $1-1 /(2 N)$, with $N$ being defined as the number of trials, to prevent an infinite d-prime (Macmillian and Creelman, 2004). Repeated measures ANOVA and paired t-tests were used to test for differences as described in the Results section. Analyses were conducted using MATLAB (MathWorks; RRID:SCR_ 001622).

450

451

452

453

454

455

456

457

458

459

460

461

462

463

464

465

466

467

468

\section{$\underline{\text { Results }}$}

\section{Behavioral Results}

To determine the effects of reward and cue visibility on sequence monitoring dynamics in the RLPFC, we used a sequence monitoring task (based on (Desrochers et al., 2019) with value and visibility manipulations to create a two-by-two design (FIG. 1). Additionally, to separate processes related to sequence and reward, reward was provided after multiple sequences were performed (rather than one). Here, we use the term "iteration" to indicate one instance of the ordered image set, or one four-item sequence. In other words, rather than reward being associated with one iteration (as is typical), reward was provided after multiple sequence iterations, at the end of the block. During each block, participants monitored the sequential order of visible or internally tracked (occluded) items that were members of high or low value sequences.

We first assessed reaction time (RT) on sequence monitoring trials (prior to the final probe trial) using a repeated measures ANOVA (rmANOVA) including factors for visibility (visible/occluded), reward (high/low), and position (first position/subsequent positions (2-4)). We replicated previous observations (Desrochers et al., 2015, 2019) that the RT at the first position in the sequence was slowed with respect to the subsequent positions in the sequence (2-4) (rmANOVA, position: $F(1,35)=25.7, p<0.001 ; \eta_{p}^{2}=0.42 ;$ FIG 3A). This 
469 sequence initiation cost provides evidence that participants were monitoring the items as 470 sequences (Schneider and Logan, 2006). Participants performed the task well (mean error 471 rate (ER), $12.5 \%$ ), and as in previous studies there was no evidence of a sequence initiation 472 cost in error rate (ER: rmANOVA, position: $F(1,35)=0.02, p=0.90, \eta_{p}{ }^{2}=0.001$; FIG 3B).

473

474

475

476

477

478

479

480

481

482

483

484

485

486

487

488

489

490

491

492

493

494

495

496

497

498

499

500

501

502

503

504

505

506

507

508

509

510

511
Next, we assessed the effect of the cue visibility manipulation, visible and occluded conditions, on task performance. During monitoring, participants were faster on occluded trials than visible trials ( $r$ ANOVA, visibility: $F(1,35)=83.9, p<0.001, \eta_{p}^{2}=0.71$; FIG 3A). This RT difference could have resulted from the explicit cue (the occluder image) that indicated no further decision was necessary on occluded monitoring trials. To test this prediction, we examined RT on probe trials, when cues were visible across both conditions. There were no reliable differences between the visible and occluded conditions on probe trials ( $r$ ANOVA, visibility: $F(1,35)=0.12, p=0.73, \eta_{p}^{2}=0.003$ ), and we again replicated slower RT for the first relative to other positions in the sequence (rmANOVA, position: $F(1,35)=15.3, p<0.001, \eta_{p}^{2}=0.30 ; F I G 3 C$ ). Errors during monitoring trials terminated the block of trials, and happened relatively infrequently (mean $0.73 \%$ of trials, across participants). Therefore, we examined ER on probe trials and found a greater ER for visible compared to occluded trials ( $r$ ANOVA, visibility: $F(1,35)=5.2, p=0.03, \eta_{p}{ }^{2}=0.16$ ). To further examine these differences in ER, we analyzed trials with respect to detecting an out of sequence item. There were no reliable differences in $d^{\prime}$ across conditions (rmANOVA: visibility, $F(1,35)=3.3, p=0.08, \eta_{p}^{2}=0.09$; reward: $F(1,35)=0.77, p=0.39, \eta_{p}^{2}=0.02$; interaction, $F(1,35)=0.008, p=0.93, \eta_{p}^{2}=0.0002$ ). There were also no reliable differences in hits (rmANOVA: F's<0.37, p's>0.54) or false alarms (rmANOVA: F's<1.01, p's $>0.32$ ). In sum, even though a difference existed in ER between occluded and visible conditions, this difference did not result in a reliable difference in the detection of out of sequence items.

To examine the effects of the reward manipulation on behavior, we compared high and low value trials. Participants responded faster on high versus low value sequences during monitoring trials ( $r$ ANOVA, reward: $F(1,35)=4.9, p=0.03, n_{p}{ }^{2}=0.12$ ) in a manner that did not interact with cue visibility (rmANOVA interaction: $F(1,35)=0.60, p=0.44, \eta_{p}^{2}=0.02$; FIG 3A). Note that while the effect of reward value on RT appears numerically small, the effect size could be interpreted as medium to large. This effect is consistent with a substantial literature illustrating faster RTs with increased reward (Niv, 2007; Guitart-Masip et al., 2011; Beierholm et al., 2013; Otto and Daw, 2019), and illustrates the efficacy of our reward manipulation. There was no effect of reward on probe trial ER (rmANOVA, reward: $\left.F(1,35)=3.6, p=0.07, \quad \eta_{p}^{2}=0.09\right)$ or any interaction between occluded and visible conditions (rmANOVA interaction: $F(1,35)=1.7, p=0.20, \eta_{p}^{2}=0.05$; FIG 3B). We also found no difference in reward for RT probe trials (rmANOVA, reward: $F(1,35)=2.7, p=0.12$, $\eta_{p}^{2}=0.07$ ) or interaction between reward and visibility conditions (rmANOVA, interaction: $F(1,35)=0.02, p=0.90, \eta_{p}^{2}=0.0004$; FIG 3C). We also confirmed that participants had learned and retained the values associated with the four image sets from the sequence preference test. Participants reliably selected the higher value image sets relative to chance during training $(\mathrm{t}(35)=45.4, \mathrm{p}<0.001)$ and after scanning $(\mathrm{t}(35)=54.1, \mathrm{p}<0.001)$. Further, 
512 there was no difference in preference between training and after scanning $(\mathrm{t}(35)=0.77, \mathrm{p}=$ $5130.45, d=0.10$ ).

\section{$514 \quad$ fMRI Results}

\section{Effect of reward on individual sequences}

516 To determine if the task engaged ramping in the RLPFC as observed in previous studies 517 (Desrochers et al., 2015, 2019), we defined a region of interest (ROI) from the parametric 518 ramping cluster in RLPFC from Desrochers et al. (2019) during the sequence monitoring 519 task (Experiment 2) on which this task is based ("D19" ROI, see Materials and Methods). In 520 the $\mathrm{D} 19 \mathrm{ROI}$, we found that parametric ramping betas were significantly different from zero 521 (t-test: $\mathrm{t}(25)=3.0, \mathrm{p}=0.005, \mathrm{~d}=0.5$; FIG 4A). Next, we examined whether variance in 522 RLPFC could be better accounted for by ramping or sustained activation. We constructed a 523 pair of models where ramp and sustain regressors competed for variance (see Materials and 524 Methods) and then examined the variance in the MR signal in RLPFC that each regressor 525 could uniquely account for (FIG 2C-D). We found that variance in the D19 ROI was better 526 accounted for by ramping, beyond what could be accounted for by sustained activation ( $\mathrm{t}$ test: $\mathrm{t}(35)=6.1, \mathrm{p}<0.001, \mathrm{~d}=2.5$ ). These results were supported by a whole-brain voxelwise contrast of parametric ramping activity across all sequence conditions. We found ramping activation in the RLPFC that extended laterally into orbitofrontal cortex, as well as ramping in visual cortex, midcingulate, and SMA (FIG 4B and TABLE 1). Thus, we replicated ramping activity during sequence monitoring with changes in reward value.

Table 1. Parametric ramp greater than baseline contrast activation values and brain areas.

\begin{tabular}{lcccccc} 
Location & $\begin{array}{c}\text { Extent } \\
\text { (voxels) }\end{array}$ & BA & $\mathbf{x}$ & $\mathbf{y}$ & $\mathbf{z}$ & $\begin{array}{c}\text { Peak } \\
\text { t-value }\end{array}$ \\
\hline Insula & 952 & N/A & -38 & 31 & -4 & 5.3 \\
L RLPFC & & $10 / 11$ & -28 & 61 & 1 & 3.92 \\
L inferior orbitofrontal cortex & & 47 & -48 & 40 & -8 & 5.21 \\
L middle frontal gyrus & & 46 & -30 & 52 & 28 & 4.59 \\
L lateral orbitofrontal cortex & & 11 & -28 & 64 & 4 & 4.36 \\
\hline L calcarine cortex & 18091 & 17 & -8 & -80 & 14 & 8.42 \\
R calcarine cortex & & 17 & 14 & -66 & 8 & 7.44 \\
L lingual gyrus & & 18 & -8 & -58 & -4 & 6.50 \\
R lingual gyrus & & 18 & 20 & -76 & -16 & 6.37 \\
L cuneus & & 19 & -6 & -84 & 42 & 6.44 \\
L superior parietal lobule & & 7 & -4 & -62 & 60 & 6.29 \\
L supramarginal gyrus & & 40 & -40 & -52 & 54 & 5.51 \\
L supramarginal gyrus & 2 & -46 & -28 & 48 & 3.48 \\
R supramarginal gyrus & & 40 & 42 & -38 & 40 & 5.03 \\
R superior occipital gyrus & & 19 & 18 & -72 & 42 & 5.26
\end{tabular}


R middle occipital gyrus

$\begin{array}{lllll}19 & 34 & -76 & 22 & 3.52\end{array}$

L fusiform gyrus

$\begin{array}{lllll}37 & -44 & -62 & -24 & 4.82\end{array}$

$\mathrm{R}$ fusiform gyrus

$\begin{array}{lllll}37 & 42 & -50 & -28 & 4.35\end{array}$

L cerebellum

$\mathrm{R}$ superior frontal gyrus

$\begin{array}{llll}\text { N/A } & -24 & -76 & -18\end{array}$

R SMA

$R$ postcentral gyrus

IFG, Triangularis

L thalamus

$\mathrm{R}$ inferior frontal (orbital)

$\mathrm{R}$ lateral orbitofrontal cortex

$\mathrm{R}$ middle frontal gyrus

$\mathrm{R}$ middle frontal gyrus

1898

9

$\begin{array}{lllll}9 & 38 & 32 & 44 & 5.23\end{array}$

$\begin{array}{llll}6 & 26 & 6 & 62\end{array}$

5.12

$\mathrm{R}$ middle cingulate

$\begin{array}{lllll}3 & 40 & -12 & 40 & 4.63\end{array}$

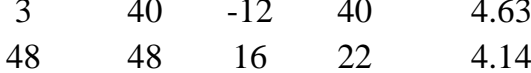

$\begin{array}{lllll}\text { N/A } & -12 & -18 & -4 & 5.04\end{array}$

$\begin{array}{lllll}47 & 36 & 28 & -8 & 5.42\end{array}$

$\begin{array}{lllll}11 & 24 & 66 & 2 & 5.02\end{array}$

$\begin{array}{lllll}46 & 42 & 52 & 12 & 3.72\end{array}$

\begin{tabular}{lll} 
& 735 & 32 \\
\hline
\end{tabular}

L SMA

$\mathrm{R}$ middle temporal gyrus

$\begin{array}{lllll}46 & 38 & 50 & 24 & 3.59\end{array}$

\begin{tabular}{llllllc}
\hline L Central Operculum & 309 & 48 & -42 & -14 & 32 & 5
\end{tabular}

L IFG, opercularis

L inferior temporal gyrus

$207 \quad 20$

All peaks are greater than $25 \mathrm{~mm}$ apart (cluster corrected $\mathrm{p}=0.05 \mathrm{FWE})$. Extent is the cluster size in voxels, listed for each peak belonging to the same cluster. BA = Brodmann's area. $x, y, z$ are MNI coordinates. IFG, inferior frontal gyrus; SMA, supplementary motor area.

Table 2. Occluded condition parametric ramp greater than baseline contrast activation values and brain areas.

\begin{tabular}{lcccccc} 
Location & $\begin{array}{c}\text { Extent } \\
(\mathbf{v o x e l s )}\end{array}$ & $\mathbf{B A}$ & $\mathbf{x}$ & $\mathbf{y}$ & $\mathbf{z}$ & $\begin{array}{c}\text { Peak } \\
\text { t-value }\end{array}$ \\
\hline R calcarine cortex & 11971 & 17 & 10 & -78 & 4 & 9.76 \\
L calcarine cortex & & 17 & -18 & -70 & 8 & 6.9 \\
L calcarine cortex & & 17 & -2 & -92 & 2 & 7.16 \\
R superior parietal lobule & & 7 & 4 & -64 & 52 & 5.63 \\
R superior parietal lobule & & 7 & 32 & -66 & 54 & 4.82 \\
L fusiform gyrus & 37 & -26 & -64 & -8 & 3.67 \\
R fusiform gyrus & & 37 & 26 & -60 & -14 & 4.8 \\
R lingual gyrus & & 18 & 8 & -48 & 2 & 4.59 \\
R ventral posterior cingulate & & 23 & 20 & -58 & 28 & 4.19 \\
\hline R inferior frontal (orbital) & 1251 & 47 & 40 & 28 & -6 & 4.91 \\
R middle temporal gyrus & & 21 & 58 & -28 & -8 & 4.81 \\
R IFG, opercularis & & 48 & 62 & -4 & 8 & 4.65 \\
\hline
\end{tabular}




\begin{tabular}{lcccccc}
\hline L middle temporal gyrus & 1046 & 21 & -48 & -4 & -12 & 5.7 \\
L superior temporal & & 48 & -48 & -24 & 10 & 4.45 \\
R IFG, opercularis & & 48 & -40 & 20 & 2 & 4.98 \\
\hline R anterior cingulate & 703 & 32 & 2 & 38 & 30 & 5.23 \\
L anterior cingulate & & 32 & -14 & 16 & 46 & 3.6 \\
R ventromedial prefrontal cortex & & 11 & 10 & 40 & 2 & 3.67 \\
\hline R RLPFC & 398 & 10 & 18 & 66 & 6 & 5.68 \\
R middle frontal orbital gyrus & & 47 & 44 & 48 & -14 & 4.54 \\
\hline L ventromedial prefrontal cortex & 558 & 11 & -12 & 34 & -16 & 5.23 \\
L RLPFC & & 10 & -22 & 64 & 4 & 5.16 \\
L inferior frontal (orbital) & & 47 & -42 & 52 & -12 & 4.76 \\
\hline R middle frontal gyrus & 376 & 9 & 30 & 36 & 38 & 4.71 \\
L supramarginal gyrus & 340 & 2 & -42 & -28 & 48 & 4.52 \\
L middle frontal gyrus & 303 & 46 & -38 & 22 & 42 & 4.56 \\
L angular gyrus & 227 & 39 & -40 & -58 & 50 & 4.05 \\
\hline All paaks arear
\end{tabular}

All peaks are greater than $25 \mathrm{~mm}$ apart (cluster corrected $\mathrm{p}=0.05 \mathrm{FWE}$ ). Extent is the cluster size in voxels, listed for each peak belonging to the same cluster. BA = Brodmann's area. $\mathrm{x}, \mathrm{y}, \mathrm{z}$ are $\mathrm{MNI}$ coordinates. IFG, inferior frontal gyrus.

To determine if monitoring without visible sequence position cues influenced RLPFC ramping in the context of changes in reward, we next examined ramping activity in the visible and occluded conditions. These analyses were performed in the parametric ramping model that contained all four (two-by-two) conditions. In the D19 ROI, although the parametric ramp regressor betas were numerically greater in the occluded than the visible condition (visible: $M=0.04$, occluded: $M=0.08$ ), there was no reliable difference between them (rmANOVA, visibility: $F(1,35)=1.2, p=0.28, \quad \eta_{p}{ }^{2}=0.03$, FIG 4A). Whole-brain voxelwise contrasts of these conditions supported these results. Though there were a greater number of areas, including the RLPFC, that survived statistical correction in the occluded condition (FIG 4C and Table 2) compared to the visible condition (FIG 4D and

Table 3), no clusters survived statistical correction in the direct contrast of these conditions. A follow up test using an ROI defined by the RLPFC cluster in the parametric ramping contrast across conditions in the current experiment (FIG 4B and Table 1) also did not show a reliable difference between ramping betas in occluded and visible conditions (rmANOVA, visibility: $F(1,35)=0.98, p=0.33, \eta_{p}^{2}=0.03$ ). These results replicate previous findings (Desrochers et al., 2019) and suggest that memory processes that are not explicitly sequential do not interact with reward-related dynamics in this task. However, these results do not rule out the possibility, and we directly test these potential interactions between reward and memory in the context of sequence monitoring dynamics in the RLPFC below.

Table 3. Visible condition parametric ramp greater than baseline contrast activation values 566 and brain areas. 
Extent Peak

\begin{tabular}{lcccccc} 
Location & (voxels) & BA & $\mathbf{x}$ & $\mathbf{y}$ & $\mathbf{z}$ & t-value \\
\hline R superior parietal lobule & 6746 & 7 & 14 & -68 & 58 & 5.86 \\
L calcarine cortex & & 17 & -12 & -80 & 12 & 5.77 \\
R calcarine cortex & & 17 & 12 & -64 & 14 & 4.98 \\
R calcarine cortex & & 17 & 12 & -64 & 14 & 4.55 \\
L lingual gyrus & & 18 & -12 & -58 & -12 & 5.61 \\
L superior occipital gyrus & & 19 & -8 & -82 & 46 & 4.13 \\
R superior occipital gyrus & 18 & 14 & -90 & 26 & 3.77 \\
L fusiform gyrus & & 37 & -34 & -74 & -16 & 4.55 \\
R fusiform gyrus & & 37 & 32 & -76 & -12 & 4.37 \\
R supramarginal gyrus & & 40 & 42 & -36 & 44 & 4.75 \\
R fusiform gyrus & & 37 & 28 & -48 & -8 & 3.55 \\
\hline L supramarginal gyrus & 528 & 40 & -40 & -50 & 52 & 5.62 \\
\hline Alpeaks ar grear
\end{tabular}

All peaks are greater than $25 \mathrm{~mm}$ apart (cluster corrected $\mathrm{p}=0.05 \mathrm{FWE}$ ). Extent is the cluster size in voxels, listed for each peak belonging to the same cluster. BA = Brodmann's area. $\mathrm{x}, \mathrm{y}, \mathrm{z}$ are $\mathrm{MNI}$ coordinates.

To test our central hypothesis that changes in reward will cause changes in individual sequence RLPFC ramping dynamics, we examined the high and low reward conditions in the same parametric ramping model. In the D19 ROI, there was no difference between parametric ramp regressor betas in the high and low reward conditions (rmANOVA, reward: $F(1,35)=0.01, p=0.91, \eta_{p}^{2}=0.002$, FIG 4A). A follow-up whole brain contrast of the parametric ramping activity in the high and low reward conditions revealed no clusters that survived statistical correction. However, this result does not exclude the possibility of an interaction such that reward may only exert an influence on individual sequence ramping dynamics in RLPFC under particular visibility conditions. Interactions between memory and other cognitive control processes have been observed in lateral prefrontal cortex (Jimura et al., 2010). In the D19 ROI there was no interaction between parametric ramp regressor beta values between the cue visibility and reward conditions (rmANOVA: F's $<1.21$, p's $>0.28$, FIG 4A). These results were also consistent with results in the RLPFC ROI defined from the current experiment parametric ramping contrast, and did not show a significant difference between ramping betas in high versus low conditions (rmANOVA, reward: $F(1,35)=0.08, p$ $=0.78, d=0.001$ ) or interaction between cue visibility and reward conditions (rmANOVA interaction: $F(1,35)=1.5, p=0.23, \eta_{p}^{2}=0.04$ ). Thus, these results did not support the hypothesis that increased reward would result in an overall increase in magnitude or change in slope in the ramping activity in the RLPFC for individual sequences (iterations). The influence of reward on sequence monitoring processes may be more complex than greater activity potentially indicative of a generally heightened state of engagement. We therefore next tested whether reward causes changes in RLPFC sequence tracking dynamics that change across sequence iterations. 


\section{Effect of reward across sequence iterations}

To test for changes across sequence iterations as a result of reward, we first examined RLPFC dynamics on a longer time scale than single sequences, i.e., across blocks of trials. Collapsing across conditions, we modeled the two complete sequence iterations of each block (iterations 2 and 3, trials 5-12) as individual trials to quantify activity in the RLPFC across the block. The first iteration of the block (trials 1-4) was excluded to avoid block initiation effects (model 5a, see Materials and Methods). In the D19 ROI, RLPFC activity appeared to increase for each sequence iteration and through the block overall (rmANOVA linear contrast: $F(1,35)=7.3, p=0.01, \eta_{p}^{2}=0.17$ ) (FIG 5A). To test if ramping activity "resets" for each sequence iteration independent of the overall increase, we detrended the activity across the block by fitting a simple linear regression across all trials 5-12 and examining the resulting residuals. Because we hypothesized that this 'reset' occurs at position 1 of each sequence iteration, we tested for differences in the first positions after detrending. In the D19 ROI, there was no difference between the first position trials in the residuals (trial 5 vs. trial 9 ; $t$-test: $t(35)=0.68, p=0.50, d=0.15$ ). Importantly, these results provide evidence that sequence specific dynamics are separable from more generalized block dynamics in the RLPFC (see also Wen et al., 2020) and provide a foundation for further examining the potential influence of memory and reward across sequence iterations.

To examine the effects of monitoring sequences without visible position cues on acrossblock dynamics in the RLPFC, we created a model that again included individual regressors for trials 5-12 and separate regressors for occluded and visible conditions (collapsed across reward conditions, model 5b, see Materials and Methods). In the D19 ROI activity, there was no interaction between trial number and visibility condition (rmANOVA interaction: $F(7,245)$ $=0.75, p=0.63, \eta_{p}^{2}=0.02$, FIG 5B). In addition, we observed a small but reliable difference between occluded and visible activity across the block (rmANOVA, visibility: $F(1,35)=4.3, p$ $=0.047, \eta_{p}{ }^{2}=0.11$, FIG 5B). This greater RLPFC activation in occluded trials across the block contrasts with results presented above when examining ramping beta values collapsed across sequence iteration. These results suggest that there is an overall increase in RLPFC activity in occluded compared to visible trials that does not change the slope of the ramping activation.

To address the central question of whether ramping activity in the RLPFC is affected by reward differently across sequence iterations, we used the same block trial number onsets model (iterations 2 and 3, trials 5-12) and included separate regressors for high and low reward conditions (across visibility conditions, model $5 \mathrm{c}$, see Materials and Methods). Across the block, we found that the difference in activity in the D19 ROI for the high and low reward conditions changed across trials such that there was a greater difference as the ending reward approached (rmANOVA interaction: $F(1,35)=2.3, p=0.02, \eta_{p}^{2}=0.06$, FIG 5C). To further examine these dynamics, we compared the slope of the lines fit to the activity for each condition in the first and second sequence iteration separately. Interestingly, we found that for the first iteration of the sequence, ramping activity in the D19 ROI for high and low reward conditions was very similar (trials 5-8; ANCOVA: $F(1,284)=0.65, p=0.42$, $\left.\eta_{p}^{2}=0.002\right)$. In contrast, ramping activity had significantly greater slope for the high 
compared to the low reward condition in the second sequence iteration (trials 9-12; ANCOVA: $\left.F(1,284)=4.8, p=0.03, \eta_{p}^{2}=0.02\right)$. Together, these results suggest that reward information, though it influences behavior throughout the block, is reflected in RLPFC activity only as the end of the block approaches. Further, these results imply that reward information is integrated into temporally extended control processes closer to points in time when the information is most relevant.

We also performed an exploratory analysis to determine if these across-block reward effects in the RLPFC changed when participants monitored the sequences with or without visual cues. For this block trial number onsets model, we constructed separate regressors for trials $5-12$, occluded, visible, high and low reward conditions (model $5 \mathrm{~d}$, see Materials and Methods). In the D19 ROI, we found no reliable interactions between reward and cue visibility conditions (rmANOVA: F's<1.13, p's $>0.34$ ). Though we cannot rule out the possibility that memory and reward processes may interact during sequence monitoring, these data do not provide evidence to support such an interaction occurs in the RLPFC.

\section{Discussion}

In this experiment, we examined the effects of reward on ramping dynamics in the RLPFC across the monitoring of multiple sequence iterations. We tested three possibilities for how changes in RLPFC dynamics might emerge: through an interaction with a memory process, as a consistent change in all individual sequences, and as a change across sequence iterations. We provide novel evidence that reward does not affect sequence tracking dynamics in the RLPFC as either a tonic increase or a change in slope consistently across individual sequences. Further, these effects do not interact with whether sequential items are monitored with visible cues. In contrast, we observed effects of reward that changed across sequence iterations, with a rapid increase in amplitude with proximity to high reward. In other brain areas and in recent computational models (Kim et al., 2020; Ott et al., 2020; Cruz and Paton, 2021; Hamid et al., 2021) this 'accelerating' impact is a hallmark of reward signaling in specific forebrain representations that are responsible for task completion, and possess the correct predictive model of ongoing events. This finding adds to prior imaging, TMS and lesion studies all indicating a distinctive role for RLPFC in sequence processing.

Similarities between blood oxygen level-dependent (BOLD) activity in the RLPFC and dopamine dynamics observed in the striatum of rodents performing temporally extended tasks (Howe et al., 2013; Hamid et al., 2015, 2021) has intriguing implications for RLPFC function. Dopamine dynamics that bifurcate only in the latter half of a sequential task between high and low rewards have been observed in the striatum of rats (Howe et al., 2013). Dopamine concentration that accelerates to reward in such studies has also been used as evidence in support of a single motivational signal for adapting to current and future behavior (Hamid et al., 2015), in contrast to separate tonic and phasic dopamine signaling (Cohen et al., 2002). Further, in subsequent studies, such dopamine signaling was only observed in situations where reward was contingent on action (i.e., instrumental) rather than not (i.e., Pavlovian), and only in brain areas that were involved in the instrumental actions 
680 (Hamid et al., 2021). Companion computational models suggest these dynamics drive credit 681 assignment specific to involved brain regions. Together, these studies suggest that the 682 RLPFC is performing an active monitoring function that integrates reward and sequence 683 information, rather than a passive observer of signals relayed from elsewhere in the brain.

684

685

686

687

688

689

690

691

692

693

694

695

696

697

698

699

700

701

702

703

704

705

706

707

708

709

710

711

712

713

714

715

716

717

718

719

720

721

722

723
An interesting qualitative observation from our data is that we found ramping dynamics specific to individual sequences and that extended throughout task blocks in RLPFC. This finding further reinforces the idea that RLPFC simultaneously processes not only local sequences, but the multi-scale sequences that are inherent in complex behavior. For example, when cooking, it is necessary not only to track the sequence of steps in what you are currently cutting up, but also to track where in the overall sequence of meal preparation steps that cutting exists. These dynamics parallel recent findings from Wen et al. (2020), who showed that the RLPFC and other 'multiple demand' regions were sensitive to stepand episode-level information and preferentially represented step (rather than task) identity. We extend these results by illustrating that reward information is also incorporated into signals in the RLPFC. Further studies are necessary to determine the mechanisms by which these control signals are multiplexed during sequences.

Our results are consistent with an integration account of RLPFC function. In non-sequential tasks, RLPFC has been shown to integrate multiple sources of task information, such as different stimulus dimensions (Nee et al., 2014), mental arithmetic operations (De Pisapia et al., 2007; de Pisapia et al., 2012), relational integration in the visuospatial (Christoff et al., 2001) or semantic domain (Bunge et al., 2005), and motivation during task updating (Bahlmann et al., 2015). These cognitive control paradigms required that context and task goals be flexibly updated during specific temporal windows dependent upon task demands. We extend these findings to sequential cognitive control by demonstrating that ramping dynamics accelerate based on temporal context, such as proximity to end reward. In this context, our results suggest that RLPFC is uniquely suited during temporally extended behaviors to integrate reward information dependent upon task relevance.

There were limitations to the present study. First, we focused on the RLPFC because, though its general role in sequential processes has been established, the specifics of how its dynamics interact with myriad other variables key to sequential control has only begun to be investigated. The RLPFC is also part of a network of areas that display ramping dynamics (FIG 4), and while an investigation of all these areas is outside the scope of the current experiment, it remains an important avenue of future research. Second, while the memory manipulation applied here showed no unique interactions and addressed potential differences in monitoring with and without external position cues, there remains the possibility that other memory processes may interact with reward (Wimmer et al., 2014; Shigemune et al., 2017) that were not explicitly tested in this experiment. For example, with visible position cues, there is likely a retrieval process that is still necessary to "check" that the cue is in the correct, remembered position. Third, we manipulated a single type of reward. Primary reinforcers such as foods, intrinsic rewards, and the valence of the reward (e.g., avoiding punishment) have potentially differing effects (Braver et al., 2014). Fourth, the 
failure to find interactions between reward value and the visibility of cues across blocks of trials should be interpreted with caution, as it is possible that we were not sufficiently powered to detect such interactions. The present work establishes a foundation on which to test these and other related variables.

In summary, this study suggests that sequential control dynamics in the RLPFC reflect an accelerating, dopamine-related reward signal in addition to both local and more extended position information. Understanding how rewards are integrated into more complex and extended timescale sequential decision processes has important implications for understanding human behavior in health and in disorders such as addiction. The multi-scale nature of these signals in the RLPFC and their interaction with dopaminergic neurons in other regions that display similar ramping dynamics will be important to examine in the future, both in human and nonhuman primate models.

\section{Figure Legends}

Figure 1. Sequence monitoring task with reward. Across all conditions, the first block starts with a red instruction screen (reward indicated by amount of dollar signs, ' $\$ \$$ ' or ' $\$$ '). Instruction images (Instr), that participants do not respond to, are serially presented to show the correct order of the current image set. Participants then respond on each monitoring trial (1 s) with an in or out of sequence button press. After the last image (probe) of the block, feedback (Feed) is given for that trial in the form of a check mark (dollar signs above to indicate reward; correct) or an ' $X$ ' for an incorrect response. A. Example visible condition block that is low value (indicated by one ' $\$$ ') and has correct feedback (check mark and ' $\$$ '). The instructed image set stimuli are visible across all monitoring trials. B. Example occluded condition (low value, same image set as in $\mathbf{A}$ ) and has error feedback (' $X$ '). The 'occluder' image is displayed for monitoring trials after instruction. Participants monitor the stimuli as if the images from the sequence are occluded by this placeholder image. Only the probe image is a member of the instructed image set. C. Hyperblock structure. A "hyperblock" is two consecutive blocks of the same condition (high value, indicated by "\$\$", illustrated here), created to maximize the number of monitoring trials (by eliminating the instruction images from the second block). The second block begins with a green screen to indicate the continuation of the same condition. Individual blocks contain multiple sequence "iterations" (Iter), defined as one four-item sequence or ordered image set. All iterations within the same block are from the same image set. D. Schematic of experimental conditions. Four image sets are randomly chosen for each participant. Each image set is only associated with either high or low value and both occluded and visible conditions. E. Example run structure. Each run contains 8 hyperblocks (16 blocks), one of each condition by image set combination such that there are two hyperblocks for each of the four conditions (visible/occluded $x$ high/low). Numbers in the schematic indicate the image set as in $\mathbf{D}$. The order of hyperblocks was counterbalanced within and across runs. Vis $=$ Visible condition. Occ $=$ Occluded condition. Solid lines with darker shades indicate the visible condition. Dashed lines with lighter shades indicate the occluded condition. Red tints and shades are high value. Blue tints and shades are low value. 
Figure 2. Example model regressors. A. Onsets were modeled separately for each position as instantaneous (zero duration) events corresponding to the sequence position onsets model (model 1; see Materials and Methods). B. Parametric ramp regressors were used for the parametric sequence position (ramping) model. Linear increase across positions 1 to 4 with instantaneous onsets (model 2). C. Sustain regressors were constructed as a square wave from the onset of position 1 to the offset (response) of position 4 (models 3 and 4). D. Ramp regressors linearly increased from the onset of the position 1 to the offset of position 4 (models 3 and 4). All regressors shown were separated by visibility and reward conditions (e.g., a model containing onsets would contain 16 regressors: Occluded High Positions 1-4; Occluded Low Positions 1-4; Visible High Positions 1-4; Visible Low Positions 1-4). Figure adapted from Desrochers et al., 2015.

Figure 3. Behavioral results. A. Mean RT across sequence position for monitoring trials. B. Mean ER across sequence position for probe trials C. Mean RT across sequence position for probe trials. SEM = standard error of the mean. Solid lines depict the visible condition and dashed lines depict the occluded condition. Red color indicates high reward and blue color indicates low reward. ${ }^{* *} \mathrm{p}<0.001$.

Figure 4. Ramping activity in $\mathrm{ROI}$ and whole-brain $\mathrm{fMRI}$ analyses. A. Mean parametric ramp regressor beta values for the $\mathrm{D} 19 \mathrm{ROI}$ in the parametric sequence position (ramping) model. Solid lines depict the visible condition and dashed lines indicate the occluded condition. B. Ramping activation shown for the parametric ramp regressor over baseline contrast in the parametric sequence position (ramping) model (see Materials and Methods). FWE cluster corrected $p=0.05$ (height $p=0.001$, extent $=191$ voxels). C. Same model as in $A$, but for the parametric ramp regressor for the occluded condition over baseline contrast (FWE cluster corrected $p=0.05$, height $p=0.001$, extent $=169$ voxels). D. Same model as in $A$ and $\mathrm{B}$, but for the parametric ramp regressor for the visible condition over baseline contrast (FWE cluster corrected $p=0.05$, height $p=0.001$, extent $=180$ voxels). Note that the direct contrast of parametric ramping in the occluded and visible conditions did not yield any clusters that survived statistical correction.

Figure 5. Activity in the D19 ROI changes across a block of trials. A. Block trial number onsets model collapsed across all conditions. B. Block trial number onsets model by visibility conditions, collapsed across reward conditions. Solid lines depict the visible condition and dashed lines indicate the occluded condition. Actual data is shown with error bars and SEM. Line fit based on sequence iteration is shown in thicker lines of the same type (solid/dashed). C. Block trial number onsets model by reward condition, collapsed across memory/visibility conditions. Red color indicates high reward and blue color indicates low reward. Actual data is shown with error bars and SEM. Line fit based on sequence iteration is shown in thick and darkened lines of the same color (red/blue). Mean percent signal change $( \pm S E M)$ from the peak (2-4 s) of the FIR. SEM = standard error of the mean. 


\section{References}

823

824

825

826

827

828

829

830

831

832

833

834

835

836

837

838

839

840

841

842

843

844

845

Bahlmann J, Aarts E, Aarts E (2015) Influence of motivation on control hierarchy in the human frontal cortex. J Neurosci 35:3207-3217.

Beck SM, Locke HS, Savine AC, Jimura K, Braver TS (2010) Primary and secondary rewards differentially modulate neural activity dynamics during working memory. PLOS ONE 5.

Beierholm U, Guitart-Masip M, Economides M, Chowdhury R, Düzel E, Dolan R, Dayan P (2013) Dopamine Modulates Reward-Related Vigor. Neuropsychopharmacology 38:1495-1503.

Botvinick M, Braver T (2015) Motivation and Cognitive Control: From Behavior to Neural Mechanism. Annu Rev Psychol 66:83-113.

Braun EK, Wimmer GE, Shohamy D (2018) Retroactive and graded prioritization of memory by reward. Nat Commun 9:4886.

Braver TS et al. (2014) Mechanisms of motivation-cognition interaction: challenges and opportunities. Cogn Affect Behav Neurosci 14:443-472.

Braver TS, Bongiolatti SR (2002) The role of frontopolar cortex in subgoal processing during working memory. Neurolmage 15:523-536.

Bunge SA, Wendelken C, Badre D, Wagner AD (2005) Analogical Reasoning and Prefrontal Cortex: Evidence for Separable Retrieval and Integration Mechanisms. Cereb Cortex 15:239-249.

Chiew KS (2021) Revisiting positive affect and reward influences on cognitive control. Curr Opin Behav Sci 39:27-33.

Chiew KS, Stanek JK, Adcock RA (2016) Reward Anticipation Dynamics during Cognitive Control and Episodic Encoding: Implications for Dopamine. Front Hum Neurosci 10. 
Christoff K, Prabhakaran V, Dorfman J, Zhao Z, Kroger JK, Holyoak KJ, Gabrieli JDE (2001) Rostrolateral Prefrontal Cortex Involvement in Relational Integration during Reasoning. Neurolmage 14:1136-1149.

Coenen VA, Schumacher LV, Kaller C, Schlaepfer TE, Reinacher PC, Egger K, Urbach H, Reisert M (2018) The anatomy of the human medial forebrain bundle: Ventral tegmental area connections to reward-associated subcortical and frontal lobe regions. Neurolmage Clin 18:770-783.

Cohen JD, Braver TS, Brown JW (2002) Computational perspectives on dopamine function in prefrontal cortex. Curr Opin Neurobiol 12:223-229.

Cruz BF, Paton JJ (2021) Dopamine gives credit where credit is due. Neuron 109:19151917.

de Pisapia N, Sandrini M, Braver TS, Cattaneo L (2012) Integration in working memory: A magnetic stimulation study on the role of left anterior prefrontal cortex. PLoS ONE $7: 1-8$.

De Pisapia N, Slomski JA, Braver TS (2007) Functional specializations in lateral prefrontal cortex associated with the integration and segregation of information in working memory. Cereb Cortex 17:993-1006.

Desrochers TM, Badre D (2012) Finding parallels in fronto-striatal organization. Trends Cogn Sci 16:407-408.

Desrochers TM, Chatham CH, Badre D (2015) The Necessity of Rostrolateral Prefrontal Cortex for Higher-Level Sequential Behavior. Neuron 87:1357-1368.

Desrochers TM, Collins AGE, Badre D (2019) Sequential control underlies robust ramping dynamics in the rostrolateral prefrontal cortex. J Neurosci 39:1060-18.

Guitart-Masip M, Beierholm UR, Dolan R, Duzel E, Dayan P (2011) Vigor in the Face of Fluctuating Rates of Reward: An Experimental Examination. J Cogn Neurosci 23:3933-3938.

Haber SN, Behrens TE (2014) The neural network underlying incentive-based learning: implications for interpreting circuit disruptions in psychiatric disorders. Neuron 83:1019-1039.

Hamid AA, Frank MJ, Moore Cl (2021) Wave-like dopamine dynamics as a mechanism for spatiotemporal credit assignment. Cell 184:2733-2749.e16.

Hamid AA, Pettibone JR, Mabrouk OS, Hetrick VL, Schmidt R, Vander Weele CM, Kennedy RT, Aragona BJ, Berke JD (2015) Mesolimbic dopamine signals the value of work. Nat Neurosci 19:117-126.

Howe MW, Tierney PL, Sandberg SG, Phillips PE, Graybiel AM (2013) Prolonged dopamine signalling in striatum signals proximity and value of distant rewards. Nature 500:575579. 
Jimura K, Locke HS, Braver TS (2010) Prefrontal cortex mediation of cognitive enhancement in rewarding motivational contexts. Proc Natl Acad Sci U S A 107:8871-8876.

Kim HGR, Malik AN, Mikhael JG, Bech P, Tsutsui-Kimura I, Sun F, Zhang Y, Li Y, WatabeUchida M, Gershman SJ, Uchida N (2020) A Unified Framework for Dopamine Signals across Timescales. Cell 183:1600-1616.e25.

Kovach CK, Daw ND, Rudrauf D, Tranel D, O'Doherty JP, Adolphs R (2012) Anterior Prefrontal Cortex Contributes to Action Selection through Tracking of Recent Reward Trends. J Neurosci 32:8434-8442.

Locke HS, Braver TS (2008) Motivational influences on cognitive control: Behavior, brain activation, and individual differences. Cogn Affect Behav Neurosci 8:99-112.

Ma L, Hyman JM, Phillips AG, Seamans JK (2014) Tracking progress toward a goal in corticostriatal ensembles. J Neurosci 34:2244-2253.

Macmillian N, Creelman C (2004) Detection theory: a user's guide., 2nd ed. Mahwah, NJ: Lawrence Erlbaum Associates.

Nee DE, Jahn A, Brown JW (2014) Prefrontal cortex organization: dissociating effects of temporal abstraction, relational abstraction, and integration with FMRI. Cereb Cortex 24:2377-2387.

Niv Y (2007) Cost, Benefit, Tonic, Phasic: What Do Response Rates Tell Us about Dopamine and Motivation? Ann N Y Acad Sci 1104:357-376.

Ott F, Marković D, Strobel A, Kiebel SJ (2020) Dynamic integration of forward planning and heuristic preferences during multiple goal pursuit. PLoS Comput Biol 16:1-27.

Otto AR, Daw ND (2019) The opportunity cost of time modulates cognitive effort. Neuropsychologia 123:92-105.

Pochon JB, Levy R, Fossati P, Lehericy S, Poline JB, Pillon B, Le Bihan D, Dubois B (2002) The neural system that bridges reward and cognition in humans: an fMRI study. Proc Natl Acad Sci U S A 99:5669-5674.

Schneider DW, Logan GD (2006) Hierarchical control of cognitive processes: Switching tasks in sequences. J Exp Psychol Gen 135:623-640.

Shallice T, Fletcher P, Frith CD, Grasby P, Frackowiak RSJ, Dolan RJ (1994) Brain regions associated with acquisition and retrieval of verbal episodic memory. Nature 368:633635.

Shigemune Y, Tsukiura T, Nouchi R, Kambara T, Kawashima R (2017) Neural mechanisms underlying the reward-related enhancement of motivation when remembering episodic memories with high difficulty. Hum Brain Mapp 38:3428-3443.

Thiebaut de Schotten M, Dell'Acqua F, Valabregue R, Catani M (2012) Monkey to human comparative anatomy of the frontal lobe association tracts. Cortex 48:82-96. 
920 Totah NK, Kim Y, Moghaddam B (2013) Distinct prestimulus and poststimulus activation of VTA neurons correlates with stimulus detection. J Neurophysiol 110:75-85.

922 Van Essen DC, Glasser MF, Dierker DL, Harwell J (2012) Cortical parcellations of the macaque monkey analyzed on surface-based atlases. Cereb Cortex 22:2227-2240.

Wang, J., Narain, D., Hosseini, E.A. et al. Flexible timing by temporal scaling of cortical responses. Nat Neurosci 21, 102-110 (2018). https://doi.org/10.1038/s41593-0170028-6.

Wen T, Duncan J, Mitchell DJ (2020) Hierarchical representation of multistep tasks in multiple-demand and default mode networks. J Neurosci 40:7724-7738.

Wimmer GE, Braun EK, Daw ND, Shohamy D (2014) Episodic Memory Encoding Interferes with Reward Learning and Decreases Striatal Prediction Errors.

Yee DM, Braver TS (2018) Interactions of motivation and cognitive control. Curr Opin Behav Sci. 
a Visible

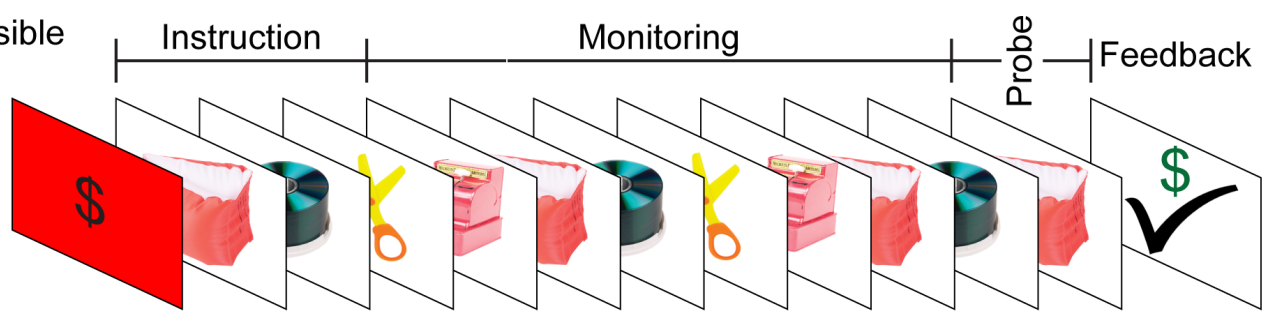

b Occluded
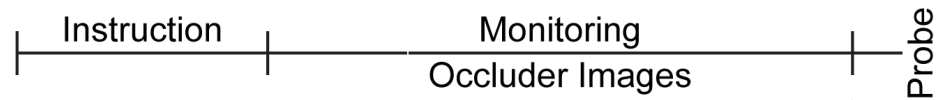
은 Feedback

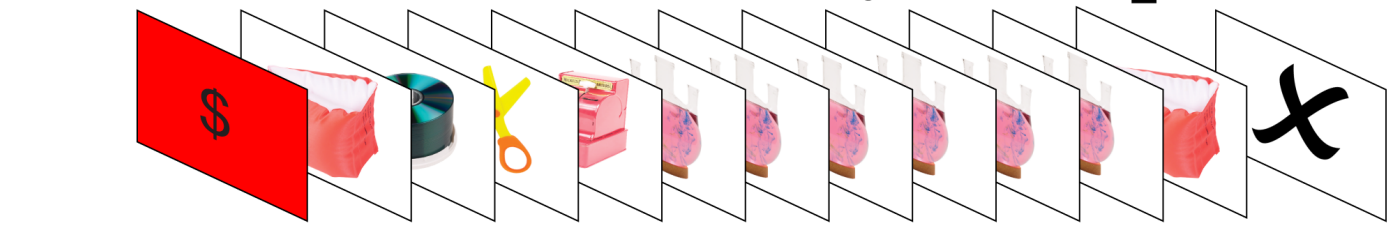

C Hyperblock

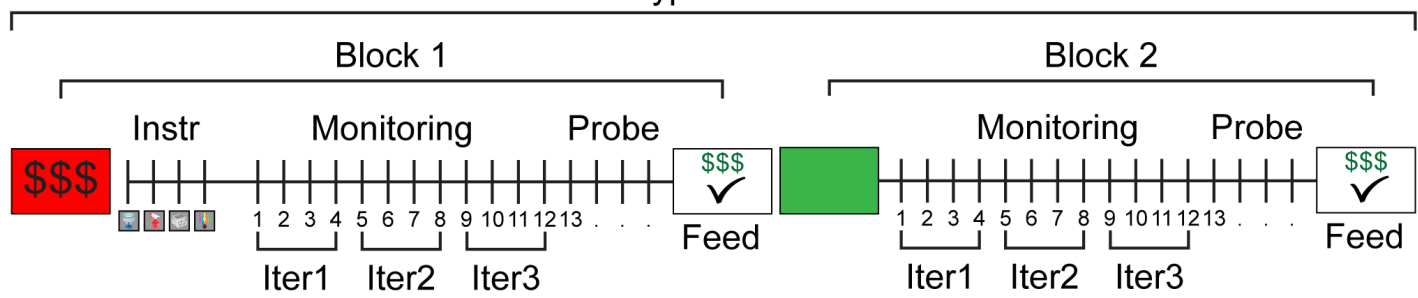

d
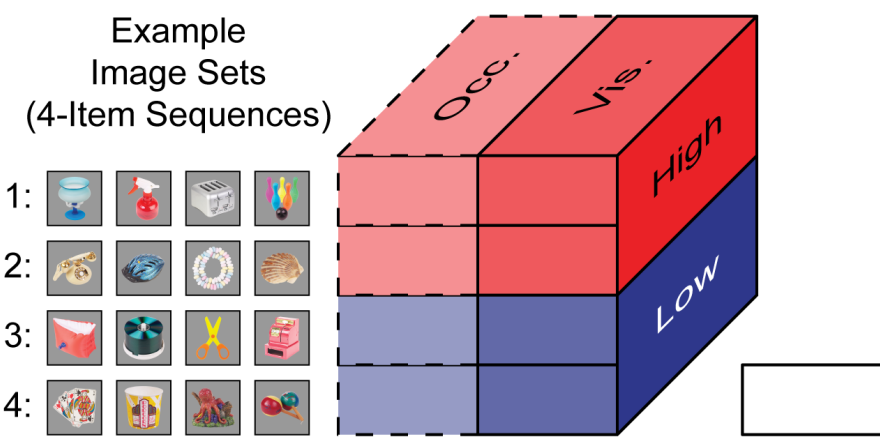
= 1 Hyperblock

e Example Run with Image Set Indicated

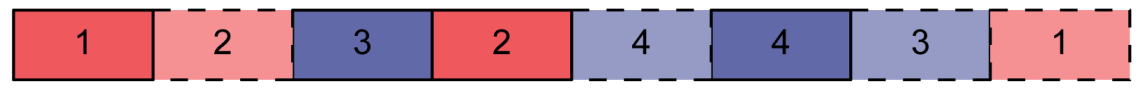

Figure 1 


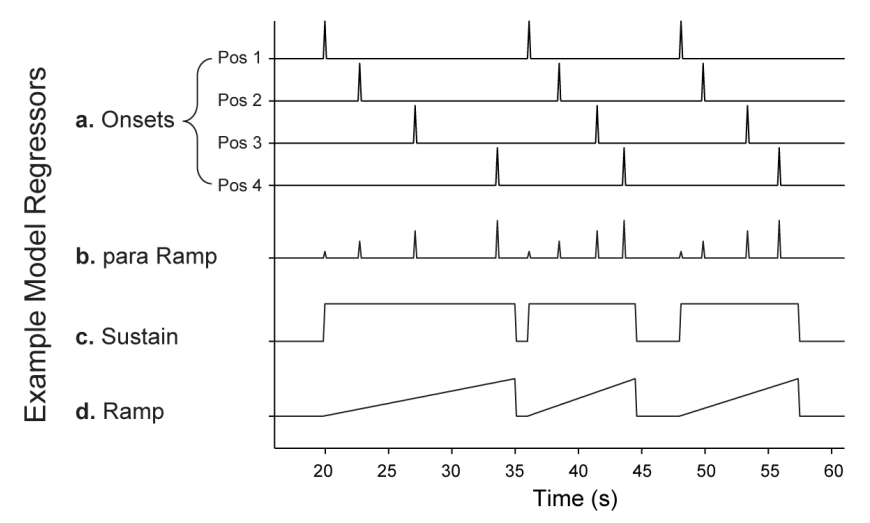

0

단

(v)

ก

5

E

10

$\sum$

(O)

(1)

느

Q

()

(U)

(U)

$<5$

(2)

는

5

(d)

(d)

Figure 2 

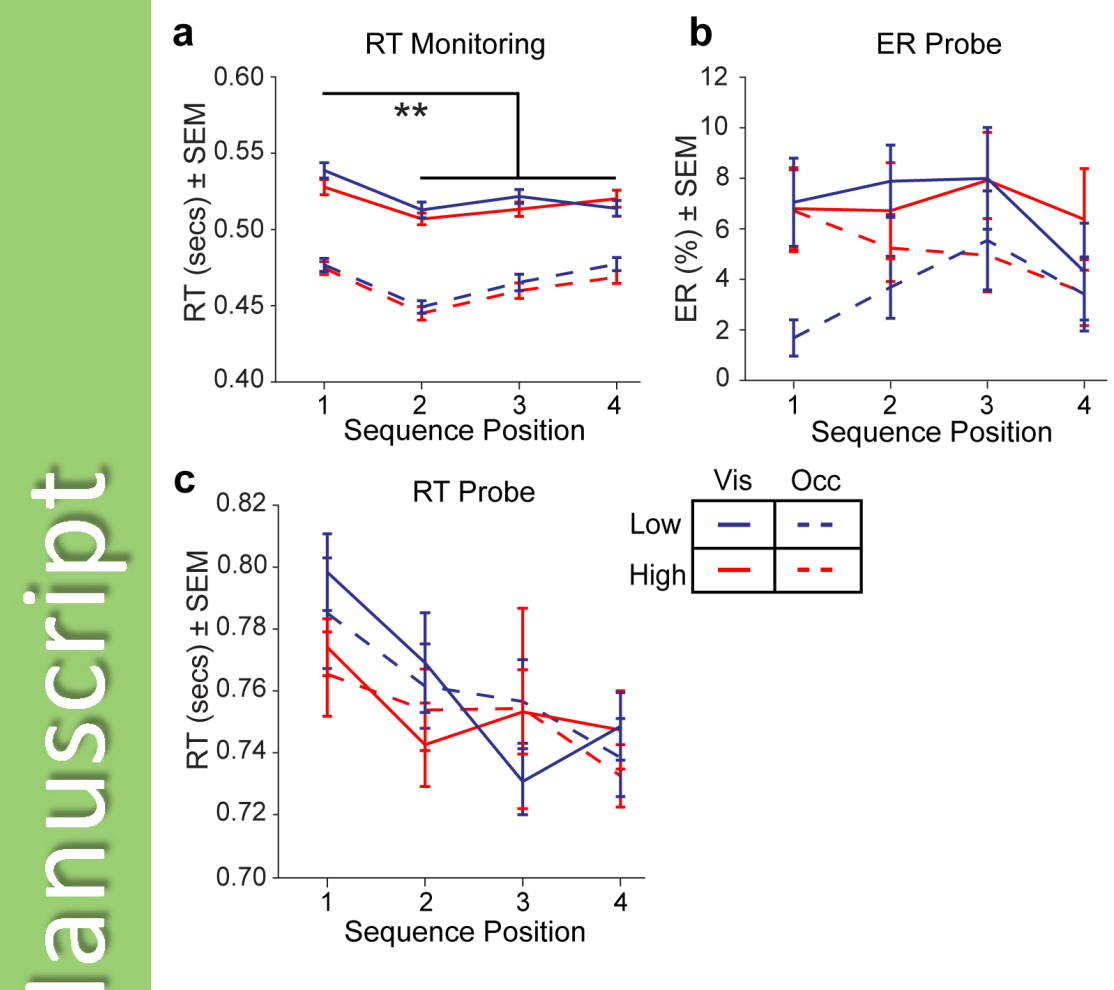

\begin{tabular}{c|c|c|}
\multicolumn{1}{r}{ Vis } & \multicolumn{1}{c}{ Occ } \\
\cline { 2 - 3 } Low & - & -- \\
\cline { 2 - 3 } High & - & -- \\
\cline { 2 - 3 } & &
\end{tabular}

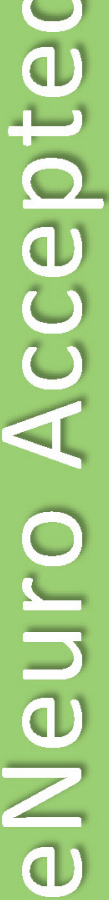

Figure 3 


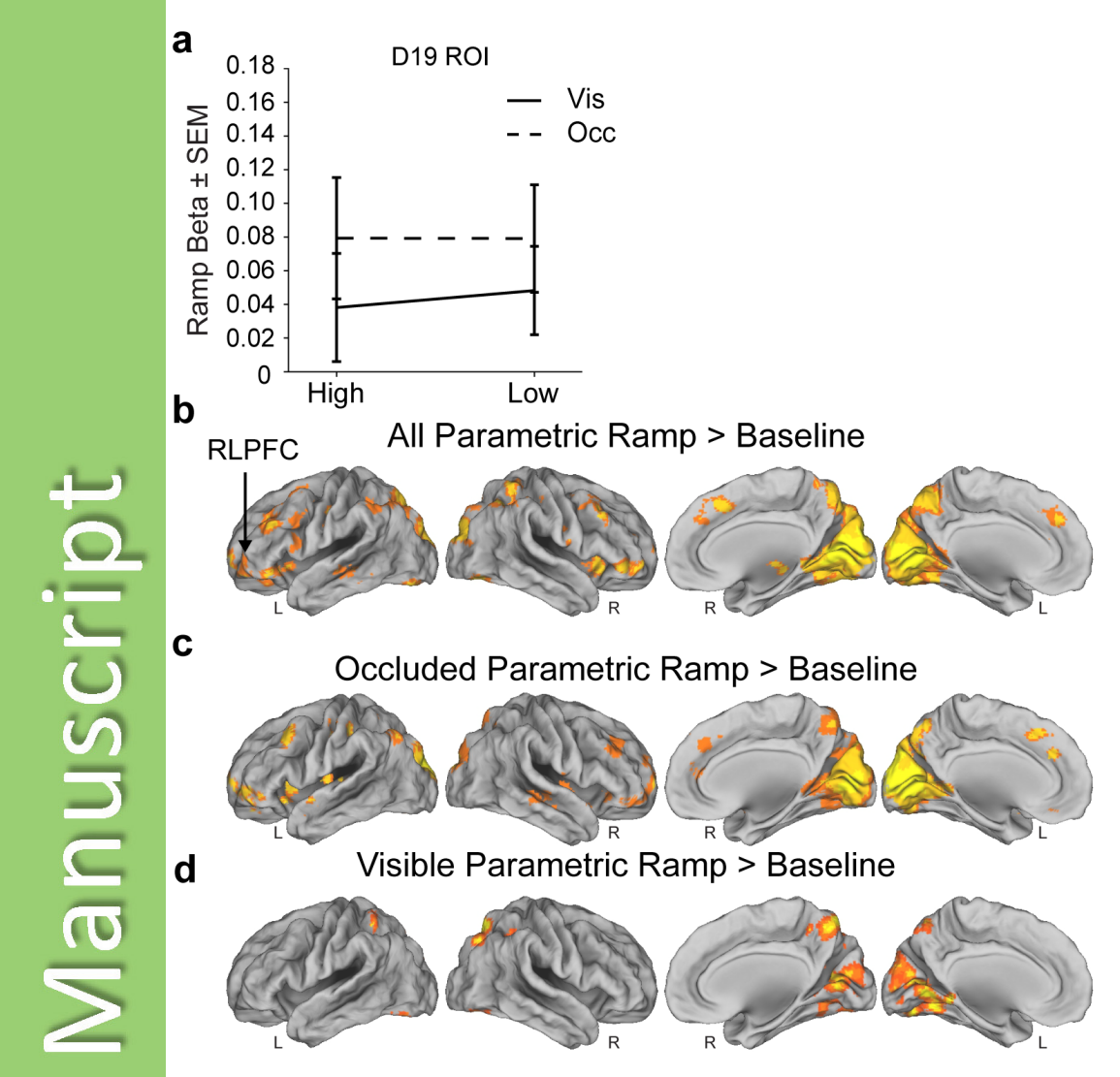

0
(1)
a
(1)
u
$\frac{1}{0}$
2
(1)
(1)

Figure 4 


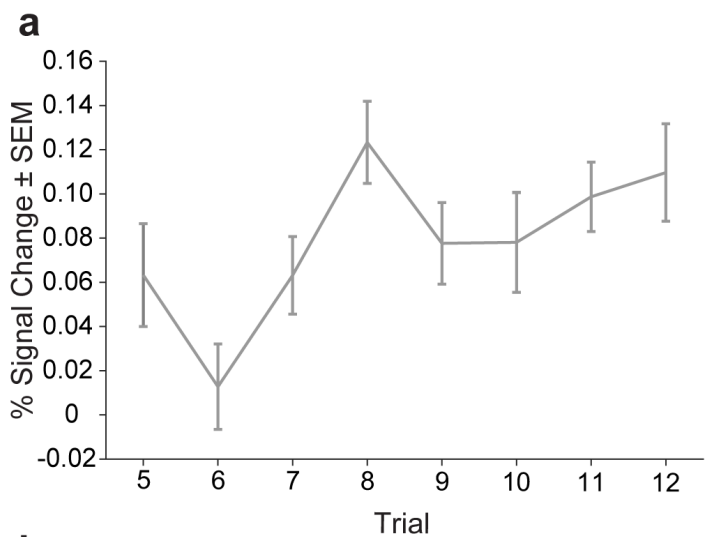

b

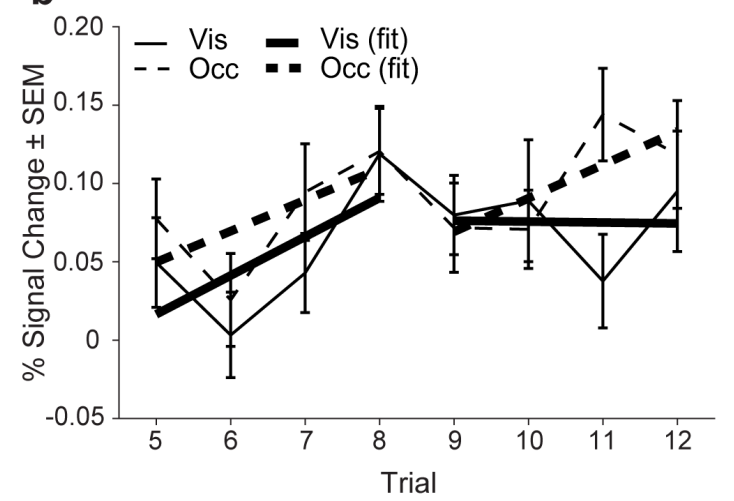

C

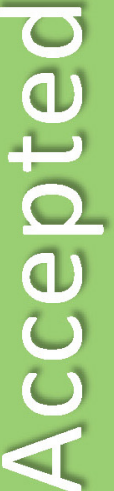

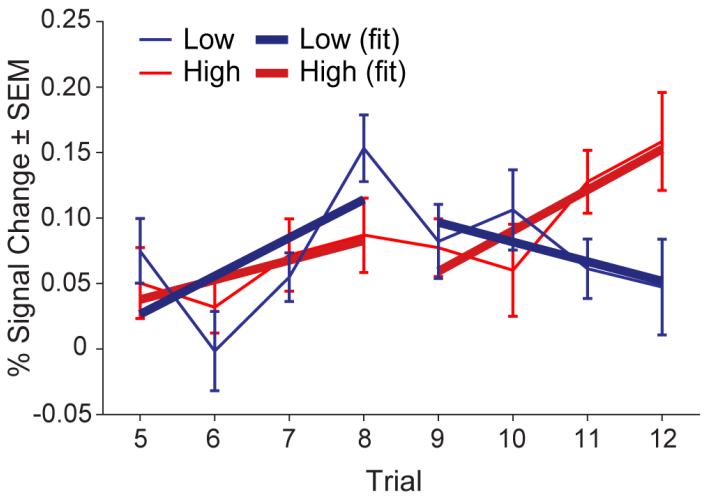

(อ)

Figure 5 